\title{
Present trends in bariatric surgery in Poland
}

\author{
Maciej Walędziak ${ }^{1}$, Anna M. Różańska-Walędziak², Piotr K. Kowalewski ${ }^{1}$, Michal R. Janik ${ }^{1}$, Jakub Brągoszewski ${ }^{1}$, \\ Krzysztof Paśnik ${ }^{1}$, Grzegorz Bednarczyk³ , Grzegorz Wallner ${ }^{4}$, Maciej Matłok ${ }^{3}$ \\ ${ }^{1}$ Department of General, Oncological, Metabolic and Thoracic Surgery, Military Institute of Medicine, Warsaw, Poland \\ ${ }^{2} 2^{\text {nd }}$ Department of Obstetrics and Gynecology, Medical University of Warsaw, Warsaw, Poland \\ ${ }^{3}$ Department of Endoscopic and Metabolic Surgery, Pro-Familia Specialized Hospital, Rzeszow, Poland \\ ${ }^{4} 2^{\text {nd }}$ Department of General, Gastrointestinal and Surgical Oncology, Alimentary Tract, Medical University of Lublin, Lublin, Poland
}

Videosurgery Miniinv 2019; 14 (1): 86-89

DOI: https://doi.org/10.5114/wiitm.2018.77707

\begin{abstract}
Introduction: As obesity has become a major health problem in Poland and bariatric procedures are the best way of treatment, an increasing trend has been observed in Polish bariatric surgery for the last decade.

Aim: Our purpose was to provide an updated overview of the bariatric surgical procedures performed in Polish institutions in comparison to the situation in Europe as well as to analyze the trends in Polish bariatric surgery over the last decades.

Material and methods: A questionnaire about the number and type of bariatric procedures performed in 2016 was sent to all Polish surgical departments. Two hundred and sixty surgical departments returned the questionnaires.

Results: Twenty-seven departments reported having performed bariatric operative or endoscopic procedures in 2016. The total number of procedures reported was 1958, the most popular being the laparoscopic sleeve gastrectomy (LSG). More than 99\% of procedures were performed using laparoscopic techniques. The most common operations were: LSG $(64.6 \%, n=1032)$ and laparoscopic Roux-en-Y gastric bypass (LRYGB) (18.2\%; $n=291)$, followed by one anastomosis gastric bypass (OAGB) (8.3\%; $n=132)$ and laparoscopic adjustable gastric banding (LAGB) $(7.3 \% ; n=117)$.

Conclusions: Registers of bariatric procedures provide information that helps in planning treatment and predicting possible complications. Adequate reporting of bariatric procedures is necessary to present the importance of the high incidence of obesity and the importance of its treatment. To collect reliable data, a national Polish bariatric surgery registry should be created.
\end{abstract}

Key words: obesity, bariatric/metabolic surgery, endoluminal procedures.

\section{Introduction}

According to the World Health Organization's (WHO) data from 2016, 39.3\% of adults in Poland are overweight and $23.1 \%$ are obese. The percentage of the overweight and obese population in Poland has doubled over the last 40 years, similarly to other developed countries [1]. According to the International Federation for the Surgery of Obesity and Metabolic Disorders (IFSO), over 600000 procedures were per- formed in 2014 in the 56 countries that are members of the IFSO, 20 of which have national bariatric registers. Since a Polish registry is currently under development, the collected data need to be based on voluntary questionnaires. As Polish bariatric surgery surveys results were previously published in 2007 by Janik et al. [2] and in 2015 by Stanowski et al. [3], we wanted to present the most recent results of the survey from Polish bariatric centers as

\section{Address for correspondence}

Maciej Walędziak MD, Department of General, Oncological, Metabolic and Thoracic Surgery, Military Institute of Medicine,

128 Szaserow St, 00-141 Warsaw, Poland, phone: +48 606387 636, e-mail: maciej.waledziak@gmail.com 
the data regarding Poland in the latest IFSO report seem to be inaccurate.

\section{Aim}

Our purpose was to report an updated overview of the bariatric surgical procedures performed in Polish institutions in comparison to the situation in Europe as well as to analyze the trends in bariatric surgery over the last decades. We also tried to investigate whether the Polish trends are similar to the global ones.

\section{Material and methods}

A questionnaire evaluating the number and characteristics of bariatric procedures performed in 2016 was sent to all Polish surgical departments. Two hundred and sixty surgical departments returned the questionnaires, 27 of which reported having performed bariatric operative or endoscopic procedures in 2016.

\section{Results}

The total number of bariatric procedures performed in Poland in 2016 included 1958 operations and 49 endoscopic procedures. Due to incoherence in the reports, only 1597 procedures could be classified, since 361 had been reported as unknown type (18.4\%). Among $82.5 \%$, the most common operations were: LSG (64.6\%,

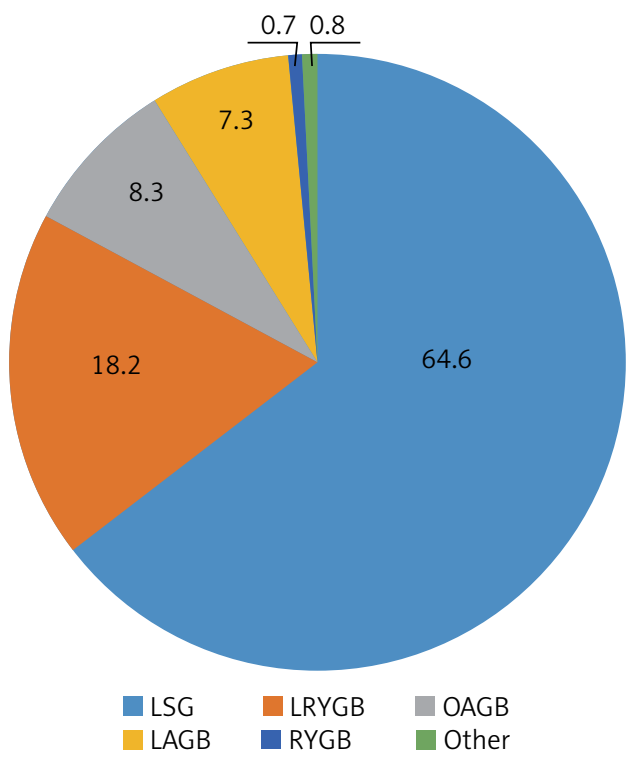

Figure 1. Percentage of surgical procedures performed in Poland in 2016 $n=1032)$ and LRYGB (18.2\%; $n=291)$, followed by one anastomosis gastric bypass (OAGB) $(8.3 \% ; n=132)$ and LAGB $(7.3 \% ; n=117)$. According to the data gathered, the number of open approach bariatric procedures has been decreasing, although it is still performed, as 12 $(0.7 \%)$ cases of RYGB were reported. Among the remaining $13(0.8 \%)$ procedures, there were 3 cases of single anastomosis duodeno-ileal bypass with sleeve gastrectomy (SADI-S) $(0.2 \%, n=3)$, for the first time reported in the Polish survey (Figure 1).

Thirteen centers reported performing more than 50 bariatric procedures a year, 4 between 100 and 200, and two departments (Warsaw and Gdansk) performed more than 300 operations. In these two main bariatric centers, the most common operation was sleeve gastrectomy (accordingly $57 \%$ and $55 \%$ ).

Among 21 surgical departments that had presented the distribution of their operations, 18 centers reported performing LSG and 10 LRYGB. Two bariatric centers performed MGB, another two LAGB, and only one center SADI. Four departments perform endoscopic procedures. Two departments reported open approach bariatric operations.

\section{Discussion}

High effectiveness and safety of bariatric procedures positively influence the patients' quality of life [4-7]. The implementation of the ERAS protocol in bariatric surgery [8] helped to increase the popularity of surgical treatment of obesity, which is the trend presented in our survey.

The number of bariatric procedures in Poland continues to increase every year, from 176 in 2007, 841 in 2012, 1499 in 2014, up to 1958 procedures in $2016[2,3]$ (Figure 2).

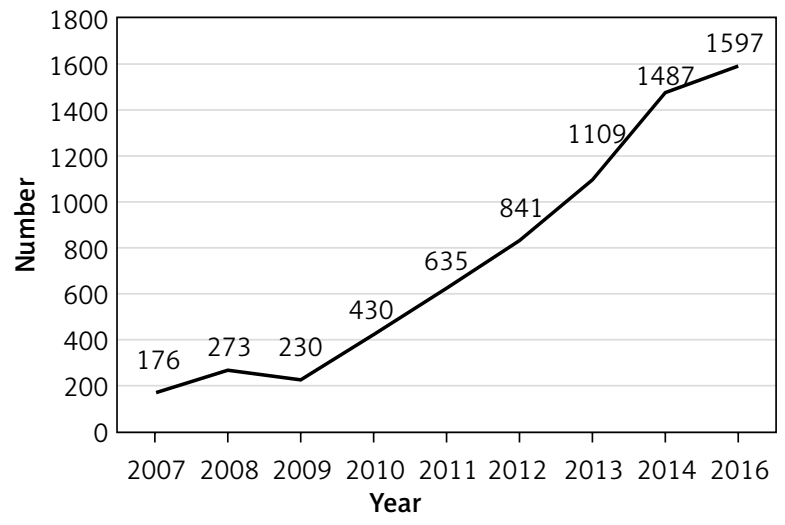

Figure 2. The number of bariatric procedures in Poland in 2007-2016 


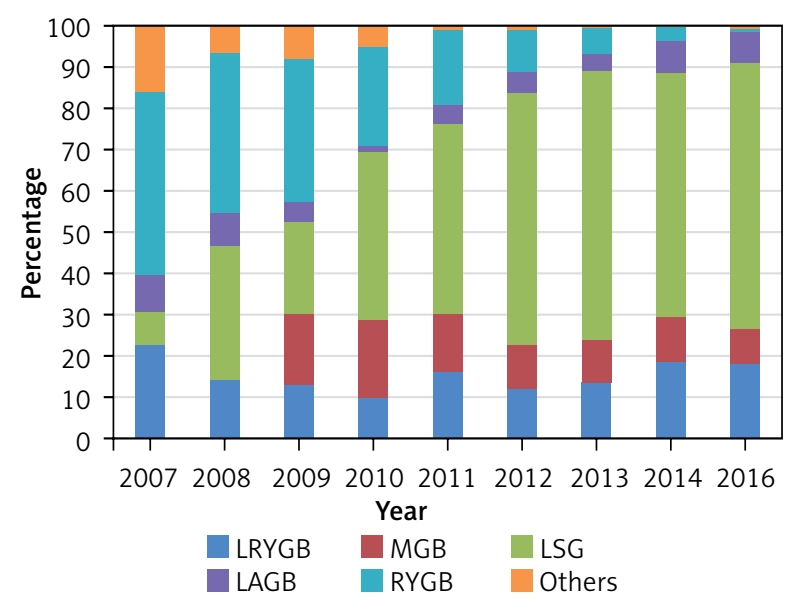

Figure 3. Trends in bariatric surgery in Poland in 2017-2016

Laparoscopy, which tends to be a method of choice in bariatric surgery, dominates, with more than $99 \%$ of procedures. Open surgery constitutes less than $1 \%$ of all operations, which is in accordance with the global trend, as stated by the IFSO report from 2017 (Third IFSO Global Registry Report 2017), 98.8\% of bariatric procedures having been performed by laparoscopy [9].

There were 49 procedures performed with endoscopic techniques, for the first time reported by any Polish bariatric centers. According to Żurawiński et al., endoscopic intragastric balloon implantation is an effective and safe method of excess body mass reduction in patients with morbid obesity before a planned bariatric surgical procedure [10]. The decreasing trend in open bariatric surgery had already been observed by Janik et al. [2]. In 2008, 106 out of 273 bariatric procedures (38.8\%) were open abdominal operations, compared to $3.6 \%$ in 2014 and less than 1\% in 2016 (Figure 3).

Laparoscopic sleeve gastrectomy continues to outnumber the rest of procedures with 879 (58.6\%) procedures in 2014 and 1032 (64.6\%) in 2016 and remains the most popular bariatric procedure in Poland.

The percentage of LRYGB operations has not changed - 18.5\% in 2014 and $18.2 \%$ in 2016. According to the survey presented by Janik et al., there was an increasing tendency in the number of LAGB procedures from 2010 to 2014, when 114 (7\%) LAGB operations were performed. The 2016 survey presents a stable number of 117 (7\%) reported LAGB procedures, despite poor long-term outcomes reported by the Polish bariatric centers [11, 12] (Figure 3).
Polish trends tend to be similar to international ones. According to the 2014 survey, the results of which were published by Angrisani et al., sleeve gastrectomy was the most common bariatric procedure in the world [13]. Sleeve gastrectomy constituted $45.9 \%$ of the bariatric operations in the world in 2014 compared to $58.6 \%$ in 2014 and $64.6 \%$ in 2016 in Poland. $39.6 \%$ of operations worldwide were LRYGB, whereas in Poland the percentage was only $18.5 \%$ in 2014 and $18.2 \%$ in 2016 . Nevertheless, the annual percentage of sleeve gastrectomies continues to increase in the IFSO regions and in Poland.

The major limitation of this study was the problem with gathering reliable data. Several surgical departments did not follow the principles of the questionnaire and they did not report the precise numbers of procedures performed. Out of 1958 operations, only 1597 were included in the study, since 361 (18.4\%) procedures had been reported without a declared type. This could be omitted in future surveys if a national registry of bariatric operations is created. There have been many attempts to create such a registry that would allow gathering of reliable data about types of operations, bariatric surgeons and complications, but unfortunately none of them has been successful so far in Poland. Only 259 bariatric procedures performed by 3 Polish surgical centers were reported to the IFSO register of more than 200000 operations performed globally from 2013 to 2017 [9].

\section{Conclusions}

Registers of bariatric procedures provide information that helps in planning treatment and predicting possible complications. Adequate reporting of bariatric procedures is necessary to present the importance of the high incidence of obesity and the importance of its treatment. To collect reliable data, a national Polish bariatric surgery registry should be created.

\section{Conflict of interest}

The authors declare no conflict of interest.

\section{References}

1. WHO, Global Health Observatory data repository, Overweight/ Obesity: http://apps.who.int/gho/data/node.main.A896?lang=en 2. Janik MR, Stanowski E, Paśnik K. Present status of bariatric surgery in Poland. Videosurgery Miniinv 2016; 11: 22-5. 
3. Stanowski E, Wyleżoł M, Paśnik K. Laparoscopy in bariatric surgery in Poland - present status. Videosurgery Miniinv 2007; 2: 18-23.

4. Paluszkiewicz R, Kalinowski P, Wróblewski T, et al. Prospective randomized clinical trial of laparoscopic sleeve gastrectomy versus open Roux-en-Y gastric bypass for the management of patients with morbid obesity. Videosurgery Miniinv 2012; 7 : 225-32.

5. Kowalewski PK, Olszewski R, Walędziak M, et al. Long-term outcomes of laparoscopic sleeve gastrectomy - a single-center, retrospective study. Obes Surg 2018; 28: 130-4.

6. Major P, Matłok M, Pędziwiatr M, et al. Quality of life after bariatric surgery. Obes Surg 2015; 25: 1703-10.

7. Janik MR, Rogula T, Bielecka I, et al. Quality of life and bariatric surgery: cross-sectional study and analysis of factors influencing outcome. Obes Surg 2016; 26: 2849-55.

8. Major P, Stefura T, Małczak P, et al. Postoperative care and functional recovery after laparoscopic sleeve gastrectomy vs. laparoscopic Roux-en-Y gastric bypass among patients under ERAS protocol. Obes Surg 2018; 28: 1031-9.

9. Higa K, Himpens J, Welbourn R, et al. Third IFSO Global Registry Report 2017. IFSO \& Dendrite Clinical Systems Ltd., Henley-onThames, Oxfordshire, UK 2017.

10. Żurawiński W, Sokołowski D, Krupa-Kotara K, et al. Evaluation of the results of treatment of morbid obesity by the endoscopic intragastric balloon implantation method. Videosurgery Miniinv 2017; 12: 37-48.

11. Kowalewski PK, Olszewski R, Kwiatkowski AP, Paśnik K. Revisional bariatric surgery after failed laparoscopic adjustable gastric banding - a single-center, long-term retrospective study. Videosurgery Miniinv 2017; 12: 32-6.

12. Kowalewski PK, Olszewski R, Kwiatkowski A, et al. Life with a gastric band. Long-term outcomes of laparoscopic adjustable gastric banding - a retrospective study. Obes Surg 2017; 27: 1250-3.

13. Angrisani L, Santonicola A, lovino P, et al. Bariatric surgery and endoluminal procedures: IFSO Worldwide Survey 2014. Obes Surg 2015; 25: 1822-32.

Received: 29.03.2018, accepted: 28.06.2018. 